\title{
A Meta-Analysis of Deceptive Advertising: An Abstract
}

\author{
Pamela A. Richardson-Greenfield
}

\begin{abstract}
Deceptive advertising has been researched in the context of marketing, business, psychology, economics, law, public policy, health, food, and communications. Many studies document a negative relationship between deceptive advertising and consumer evaluations, such as attitudes toward the brand and ad, trustworthiness, purchase intention, and likelihood to recommend. For instance, Cowley (2006) found that puffery claims are perceived as less credible by consumers than factual claims, Boyer et al. (2015) discovered that covert marketing elicits more negative attitudes toward a product than overt marketing, and Craig et al. (2012) showed that highly deceptive ads are associated with lower purchase intentions than are factual ads. However, some forms of deceptive advertising do not produce adverse effects. For example, Xu and Wyer (2010) found that puffery actually was related to higher consumer product evaluations. Further, misleading claims have been shown to relate to more positive consumer attitudes toward ads and brands (Newell et al. 1998), and greenwashing is believed to have a positive impact on brand imagery (Parguel et al. 2015).

The current study seeks to summarize the deceptive advertising research by conducting a meta-analysis of this stream of literature over a 35-year period. Metaanalysis is a practical methodological approach that assesses the generalizability of relationships more objectively than narrative reviews and can identify relationships and boundary conditions that have not been - and could not be-assessed in a single empirical study (Brown et al. 1998). Specifically, we employ meta-analysis to examine the effects of deceptive advertising on consumer evaluations and identify important moderators of this relationship including the type of deception, the type of advertisement (e.g., print, TV commercial), and the focal products being evaluated, in addition to study design features. Our aim is to synthesize the existing research findings concerning deceptive advertising and consumer evaluations, especially in terms of the relevant moderators identified above (Jaramillo et al. 2005).
\end{abstract}

\footnotetext{
P. A. Richardson-Greenfield $(\bowtie)$

University of Texas at Arlington, Arlington, TX, USA

e-mail: pamelaarichardson@mavs.uta.edu
} 